\title{
ORIGINAL RESEARCH \\ Diagnostic Yield of Double-Dose Gadobutrol in the Detection of Brain Metastasis: Intraindividual Comparison with Double-Dose Gadopentetate Dimeglumine
}

E.S. Kim

J.H. Chang

H.S. Choi

J. Kim

S.-K. Lee

BACKGROUND AND PURPOSE: Accurate assessment of the number and lesion characteristics of brain metastasis is very important in GKS. The purpose of this study was to compare the diagnostic efficacy of DD gadobutrol in the detection of brain metastases compared with a DD $0.5-\mathrm{mol} / \mathrm{L}$ gadolinium contrast, gadopentetate dimeglumine.

MATERIALS AND METHODS: Records of 27 patients (male to female ratio, 15:12; mean age, 57.1 years) diagnosed with brain metastasis and having undergone GKS were retrospectively analyzed. All patients underwent the first 3D-T1-GRE MR imaging with a DD of gadopentetate dimeglumine. The second MR imaging with a DD of gadobutrol was performed during GKS by using the same parameters used for the first scan. Two neuroradiologists counted the number of enhancing lesions on 2 consecutive MR imaging examinations and reached consensus. Lesion-brain CNR was measured from 45 lesions, and paired $t$ test analysis was performed between DD gadopentetate dimeglumine and gadobutrol MR imaging.

RESULTS: On DD gadopentetate dimeglumine-enhanced images, a total of 130 lesions were detected visually. With DD gadobutrol, 25 additional lesions were detected on GKS MR imaging. There was no missing lesion on DD gadobutrol MR imaging. The mean lesion-brain CNR was higher on DD gadobutrol MR imaging than on DD gadopentetate dimeglumine imaging $(2.17 \pm 0.19$ versus $1.90 \pm$ 0.26; $P=.00011$, paired $t$ test, 2-tailed). Only 2 cases showed lower CNR on DD gadobutrol images: 1 with hemorrhagic metastasis from renal cell carcinoma and the other with steroid treatment after the first MR imaging.

CONCLUSIONS: DD 1.0-mol/L gadobutrol provides higher lesion conspicuity and enhances lesion detection in brain metastasis compared with DD 0.5-mol/L gadolinium contrast agents.

ABBREVIATIONS: CNR = contrast-to-noise ratio; $\mathrm{DD}=$ double dose; $\mathrm{Gd}=$ gadolinium; $\mathrm{GKS}=$ gamma knife surgery; GRE = gradient recalled-echo

$\mathbf{F}$ or therapeutic planning, it is critical to obtain the exact number and location of brain metastases. Only then can patients take advantage of recent advances in targeted radiosurgery, which has produced promising results such as higher survival rates. ${ }^{1,2}$ Compared with CT or unenhanced MR imaging, the diagnostic yield of contrast-enhanced brain MR imaging in the detection of brain metastasis is very high. ${ }^{3-5}$

Adjusting various parameters during MR imaging has been considered very important in achieving higher sensitivity in the detection of enhancing lesions or vascular structures. Among these, modification of the administered Gd dose is thought to raise the diagnostic efficacy in brain lesions. ${ }^{4,6-12}$

Among the recently available MR imaging contrast media, gadobutrol (gadolinium-DO3A-butriol, Gadovist 1.0; Schering, Berlin, Germany) is the first commercially available 1.0$\mathrm{mol} / \mathrm{L}$ gadolinium chelate, a macrocyclic chelate not a linear one. ${ }^{12-17}$ The lower osmolality and viscosity of gadobutrol enables the double-concentrated solution, which contains twice

Received September 7, 2009; accepted after revision November 21

From the Departments of Radiology (E.S.K., H.S.C., J.K., S.-K.L.) and Neurosurgery (J.H.C.), Yonsei University College of Medicine, Seoul, Korea.

Funding for this study was provided by Bayer Schering Pharma.

Please address correspondence to Seung-Koo Lee, MD, PhD, Department of Radiology, Yonsei University College of Medicine, 250 Sungsanro, Seodaemungu, Seoul, 120-752, Korea; e-mail: slee@yuhs.ac

DOI 10.3174/ajnr.A2010 the amount of Gd chelate per volume. ${ }^{12-17}$ The T1 relaxivity of gadobutrol is approximately $14 \%-27 \%$ higher than that of other $0.5-\mathrm{mol} / \mathrm{L}$ Gd chelates. ${ }^{18}$

Higher dose administration is known to be more effective in lesion detection, but consideration must be given to reducing the amount of gadolinium administered to the patient because of the risk of contrast-related reactions and nephrogenic systemic fibrosis. ${ }^{19,20}$ Gadobutrol had been proved to be a safe MR imaging contrast agent in patients with impaired renal function at doses of $\leq 0.3 \mathrm{mmol} / \mathrm{L} / \mathrm{kg}$ of weight, ${ }^{18}$ and no nephrogenic systemic fibrosis has been reported so far. ${ }^{17}$

At this point, we can postulate that DD gadobutrol is expected to offer better opportunity for further improved enhancement than other Gd contrast agents because of the higher T1 relaxation effect. However, there have been few documented data of the clinical benefits in the detection of brain disease in contrast to other $0.5-\mathrm{mol} / \mathrm{L} \mathrm{Gd}$ contrast agents. ${ }^{17}$ The purpose of this study was to compare the diagnostic efficacy of DD 1.0-mol/L gadobutrol in the detection of brain metastasis compared with DD 0.5$\mathrm{mol} / \mathrm{L}$ gadopentetate dimeglumine.

\section{Materials and Methods}

\section{Patient Population}

Retrospective analysis was performed for patients who were diagnosed with brain metastasis and had undergone GKS from December 
2008 to March 2009. In total, 27 patients (male to female ratio, 15:12; mean age, 57.1 years; range, $14-77$ years) were involved, and their primary sites were the lung $(n=22)$, kidney $(n=2)$, liver $(n=1)$, colon $(n=1)$, and rectum $(n=1)$. The study protocol was approved by the institutional review board.

\section{MR Imaging and Contrast Agent Administration}

At the outpatient oncology clinic, patients who had neurologic symptoms routinely underwent MR imaging for metastasis protocol after being injected with DD gadopentetate dimeglumine. If the patient was found to have brain metastasis and if indicated for GKS, the patient was referred to the neurosurgery department and underwent localization MR imaging for GKS after being injected with DD gadobutrol.

All examinations were performed with a 1.5T scanner (Achieva or Intera; Philips Medical Systems, Best, the Netherlands). Each patient was scanned twice with the same 3D-T1-GRE imaging after receiving an injection of DD Gd contrast agent (ie, $0.2 \mathrm{mmol} / \mathrm{L} / \mathrm{kg}$ ). The injection contained $0.2 \mathrm{~mL} / \mathrm{kg}$ of gadobutrol and $0.4 \mathrm{~mL} / \mathrm{kg}$ of gadopentetate dimeglumine because gadobutrol is a $1.0-\mathrm{mol} / \mathrm{L}$ agent and its concentration is twice as high as that of gadolinium. A mechanical injector was used with the rate of $2 \mathrm{~mL} / \mathrm{s}$ followed by a $20-\mathrm{mL}$ normal saline infusion.

An institutional MR imaging protocol for brain metastasis included routine anatomic T1/T2-weighted and GKS imaging as well as a T1-weighted 3D-GRE sequence after injection of $0.2-\mathrm{mmol} / \mathrm{L} / \mathrm{kg}$ Gd contrast agents. Axial image reconstruction was made parallel to the anterior/posterior commissure line. The parameters of T1weighted 3D-GRE imaging were as follows: TR/TE, 26/4.5 ms; flip angle, $8^{\circ}$; bandwidth, $190.8 \mathrm{~Hz}$ /pixel; $256 \times 256$ matrix with 160 - to 180 -second axis phase-encoding steps; 1 -mm section thickness; and a 25-cm FOV. Scanning time was 7 minutes 41 seconds. Post-Gd imaging was started at 4 minutes after injection of Gd contrast. The interval between 2 consecutive scannings ranged from 2 to 11 days (mean, 4.3 days).

\section{Imaging Analysis}

Two board-certified neuroradiologists (E.S.K., S.-K.L.) with $>6$ years' experience in brain MR imaging interpretation reviewed the enhanced MR images. They were fully blinded to the contrast agent used in each examination for all patients. Each blinded reader evaluated the patients' images separately and independently. They counted the number of enhancing lesions with agreement and directly compared DD gadopentetate dimeglumine- and gadobutrol-enhanced MR images.

The lesion-to-brain CNR was evaluated by using region-of-interest measurements. Regions of interest were placed within the enhancing portion of the metastatic lesions and in the contralateral normal white matter, excluding the ventricular system, to avoid partial volume effects. Care was taken to place the region of interest in an area of homogeneous contrast enhancement within the metastasis. Regions of interest were limited to the largest 3 lesions in the cases in which there were $>3$ metastatic lesions. A total of 45 lesions were measured, and the lesion-brain CNR was calculated. A paired $t$ test was used for comparison of DD gadopentetate dimeglumine- and gadobutrolenhanced MR imaging.

\section{Results}

On the DD gadopentetate dimeglumine-enhanced images, a total of 130 lesions were detected. Among 27 patients, 13 pa-

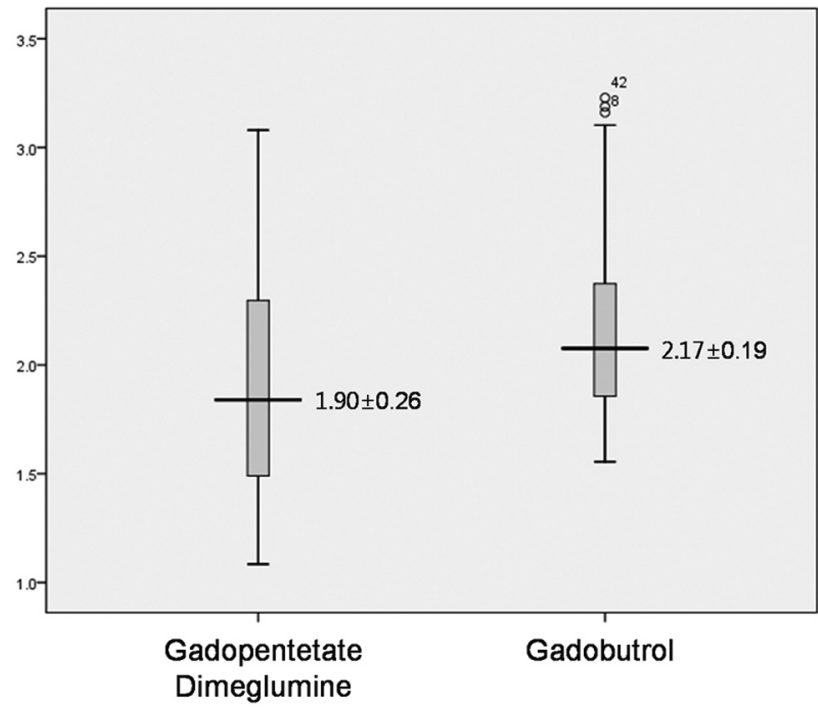

Fig 1. Comparison of lesion CNR between gadopentetate dimeglumine and gadobutrol. The mean CNR of enhancing lesions is significantly higher with gadobutrol.

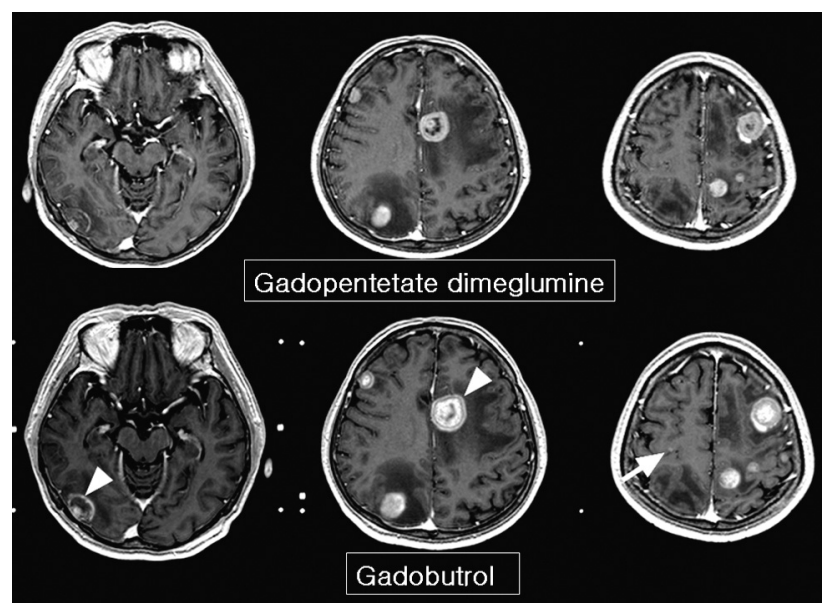

Fig 2. A 63-year-old woman with lung cancer. Gadobutrol MR imaging shows more enhancing lesions than gadopentetate dimeglumine MR imaging (arrow in the right precentral gyrus). Retrospective review of gadopentetate dimeglumine images suggests the existence of a small nodule in the right precentral gyrus, but it is not definite. A thicker enhancing rim and prominent gadolinium spillage are seen on gadobutrol MR imaging (arrowheads)

tients had a solitary lesion, 3 had 2 lesions, another 2 had 3 lesions, and 9 had multiple lesions. With DD gadobutrol, 25 additional lesions were detected on localization MR imaging for GKS in 7 patients (Fig 1). All 25 lesions were not evident on DD gadopentetate dimeglumine images. Of the 25 lesions, 1 patient had a solitary lesion, 3 had 2 lesions, 2 had 3 lesions, and 1 had 12 lesions.

The lesion-brain CNR was higher on DD gadobutrol MR imaging $(2.17 \pm 0.19$ versus $1.90 \pm 0.26 ; P=.00011$, paired $t$ test, 2-tailed) (Fig 1). Gadobutrol MR images showed thicker enhancing rims of metastatic masses (Fig 2) and better lesion contrast than gadopentetate dimeglumine images (Fig 3). Only 2 cases had lower CNR on DD gadobutrol images: 1 had hemorrhagic metastasis from renal cell carcinoma and another started steroid treatment after the first MR imaging. The CNR was 1.78 on gadobutrol MR imaging and 2.25 on gadopentetate dimeglumine MR imaging in the first patient. In the 


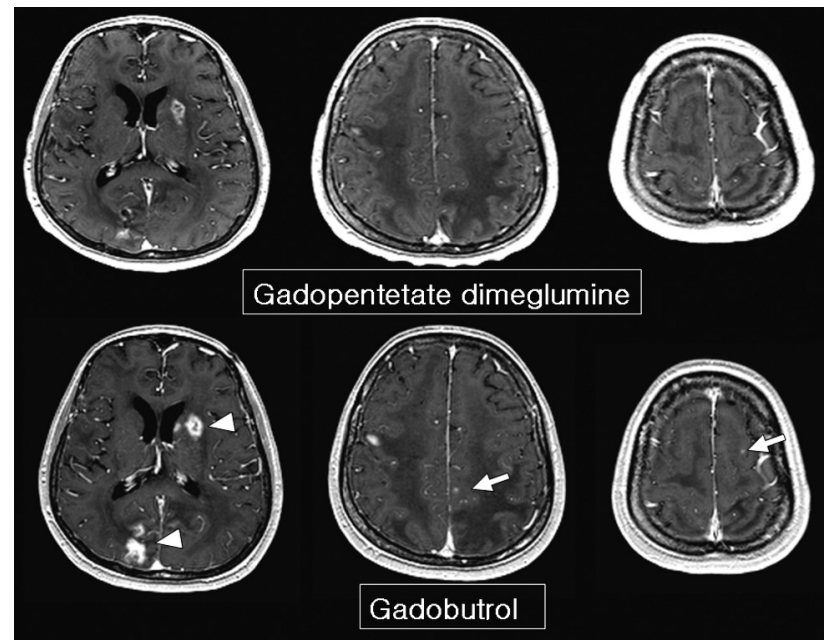

Fig 3. A 60-year-old man with lung cancer. Gadobutrol MR imaging shows more enhancing lesions than gadopentetate dimeglumine MR imaging (arrows). There is no suspected lesion on gadopentetate dimegluimine MR imaging. Note the higher signal intensity of the thick enhancing rim (arrowheads). The interval between the 2 MR images is 60 hours.

second patient, the CNR was 2.29 on gadobutrol MR imaging and 2.73 on gadopentetate dimeglumine MR imaging.

\section{Discussion}

In this study, we retrospectively analyzed the results of DD gadopentetate dimeglumine and gadobutrol in the detection of brain metastasis. Patients who showed neurologic symptoms or were suspected of having brain metastasis were administered DD gadopentetate dimeglumine in the outpatient clinic. The subjects were transferred to the neurosurgery department if they were confirmed to have hematogenous brain metastasis on MR imaging and, thus, were potential candidates for GKS. There, they received a second MR imaging for localization of metastatic lesions after setting of the gamma knife frame. It is extremely difficult for cancer patients to undergo simultaneous scanning with 2 different contrast agents. To avoid that, we decided to conduct a retrospective analysis of GKS patients as an alternative way to assess the efficacy of gadobutrol in the detection of brain metastasis.

Gadobutrol is a hydrophilic neutral macrocyclic MR imaging contrast agent with predominantly extracellular distribution in humans. ${ }^{16,18,21}$ Gadobutrol had been proved to be a safe MR imaging contrast agent in patients with impaired renal function at doses of $\leq 0.3 \mathrm{mmol} / \mathrm{L} / \mathrm{kg}$ of body weight. ${ }^{22}$ So far, there have been no reports of nephrogenic systemic fibrosis in association with the administration of gadobutrol. ${ }^{23}$ The T1 relaxivity of gadobutrol is approximately $14 \%-27 \%$ higher than that of other Gd chelates, with lower molarity, except 0.5-mol/L gadobenate dimeglumine (MultiHance; Bracco, Milan, Italy), which also has a high T1 relaxivity. ${ }^{18}$ In animal models of glioma, gadolinium concentration in the mass after gadobutrol injection is higher than that with other gadolinium chelates. ${ }^{24}$ On the basis of these previous studies, we can assume that the CNR of enhancing lesions after gadobutrol administration is much higher than that of other Gd compounds in the clinical field, but no documented data have been reported yet, to our knowledge.

Determination of the number and accurate localization of metastatic lesions is critical in GKS compared with other mo- dalities such as chemotherapy, whole brain radiation therapy, or surgery. ${ }^{25}$ Therefore, selection of excellent contrast media is very important in diagnosing brain metastasis and in the treatment plan. Because gadobutrol has been approved for clinical use by the Korean Food and Drug Administration and was commercialized the following year, neurosurgeons in our institution prefer gadobutrol for localization MR imaging because of the safety profile and higher CNR of gadobutrol; therefore, most of our localization MR imaging was performed after administration of gadobutrol.

Previous reports described the superiority of gadobutrol in contrast-enhanced MR angiography, perfusion MR imaging, and demyelinating disease. ${ }^{26-28}$ These articles reported increased diagnostic accuracy and the stable chemical and pharmacologic properties of gadobutrol, which are safer than those of previously used agents. For gadobutrol, only half the amount of the previously used $0.5-\mathrm{mol} / \mathrm{L}$ Gd contrast agents at the same concentration is enough. Thus, it is more suitable for rapid bolus injection for MR angiography and perfusion MR imaging.

A recent intraindividual comparison study by Anzalone et $\mathrm{al}^{20}$ showed the superior power of gadobutrol in the detection of brain metastasis. They analyzed 2-mm-thick 3D GRE images and determined lesion conspicuity by visual analysis. We have a similar study design, but we have more qualitative data with measurement of lesion CNR and higher resolution images with $1-\mathrm{mm}$ thickness. In routine clinical practice, DD administration with thin-section GRE imaging is common for the evaluation of metastasis. Therefore, our results have additional significance in the diagnostic accuracy of contrastenhanced MR imaging in the oncology clinic.

There are some limitations to this study. First, prospective study design and simultaneous scanning with 2 different Gd contrast agents were nearly impossible for cancer patients, and we chose a retrospective analysis. Therefore, the time interval between the $2 \mathrm{MR}$ imaging scans varied from 2 to 11 days (mean, 4.3 days). However, many enhancing lesions with 2- to 3-day intervals showed more apparent differences than lesions with longer intervals. Therefore, we postulated that these results are enough to show the higher capacity of gadobutrol in the detection of brain metastasis.

The use of a steroid is another problem. In the cases of brain edema and increased intracranial pressure, dexamethasone is usually administered, and it decreases the membrane permeability and the degree of contrast enhancement. ${ }^{29}$ Seven of 27 patients received steroid therapy during the interval between the $2 \mathrm{MR}$ imaging scans. Only 1 showed decreased lesion-brain CNR, and the others showed higher CNR with gadobutrol MR imaging. With some limitations of a retrospective study design, these results show the definite superiority of gadobutrol in diagnostic accuracy and in the detection of brain metastasis.

\section{Conclusions}

DD gadobutrol is much more effective in detecting brain metastases and provides higher lesion conspicuity in enhancing metastasis; more clinical application is expected. 


\section{References}

1. Chang SD, Lee E, Sakamoto GT, et al. Stereotactic radiosurgery in patients with multiple brain metastases. Neurosurg Focus 2000;9:e3

2. Fuentes S, Delsanti C, Metellus $P$, et al. Brainstem metastases: management using gamma knife radiosurgery. Neurosurgery 2006;58:37-42, discussion 37-42

3. Yuh WT, Tali ET, Nguyen HD, et al. The effect of contrast dose, imaging time, and lesion size in the MR detection of intracerebral metastasis. AJNR Am J Neuroradiol 1995; 16:373-80

4. Sze G, Johnson C, Kawamura Y, et al. Comparison of single- and triple-dose contrast material in the MR screening of brain metastases. AJNR Am J Neuroradiol 1998;19:821-28

5. Yuh WT, Engelken JD, Muhonen MG, et al. Experience with high-dose gadolinium MR imaging in the evaluation of brain metastases. AJNR Am J Neuroradiol 1992;13:335-45

6. Fellner F, Lungenschmid K, Fellner C, et al. Experiences with gadodiamide, a non-ionic contrast agent, in MRI of brain metastases. Rontgenpraxis 1998;51:203-11

7. Akeson P, Larsson EM, Kristoffersen DT, et al. Brain metastases: comparison of gadodiamide injection-enhanced MR imaging at standard and high dose, contrast-enhanced CT and non-contrast-enhanced MR imaging. Acta Radiol 1995;36:300-06

8. Kuhn MJ, Picozzi P, Maldjian JA, et al. Evaluation of intraaxial enhancing brain tumors on magnetic resonance imaging: intraindividual crossover comparison of gadobenate dimeglumine and gadopentetate dimeglumine for visualization and assessment, and implications for surgical intervention. J Neurosurg 2007;106:557-66

9. Rowley HA, Scialfa G, Gao PY, et al. Contrast-enhanced MR imaging of brain lesions: a large-scale intraindividual crossover comparison of gadobenate dimeglumine versus gadodiamide. AJNR Am J Neuroradiol 2008;29:1684-91

10. Tatsuno S, Hata Y, Tada S. Double-dose Gd-DTPA: detectability of intraparenchymal brain metastasis [in Japanese]. Nippon Igaku Hoshasen Gakkai Zasshi 1996;56:855-59

11. van der Molen AJ, Bellin MF. Extracellular gadolinium-based contrast media: differences in diagnostic efficacy. Eur J Radiol 2008;66:168-74

12. Vogl TJ, Friebe CE, Balzer T, et al. Diagnosis of cerebral metastasis with standard dose gadobutrol vs. a high dose protocol: intraindividual evaluation of a phase II high dose study [in German]. Radiologe 1995;35:508-16

13. Staks T, Schuhmann-Giampieri G, Frenzel T, et al. Pharmacokinetics, dose proportionality, and tolerability of gadobutrol after single intravenous injection in healthy volunteers. Invest Radiol 1994;29:709-15

14. Balzer JO, Loewe C, Davis K, et al. Safety of contrast-enhanced MR angiography employing gadobutrol $1.0 \mathrm{M}$ as contrast material. Eur Radiol 2003;13:2067-74

15. Vogler H, Platzek J, Schuhmann-Giampieri G, et al. Pre-clinical evaluation of gadobutrol: a new, neutral, extracellular contrast agent for magnetic resonance imaging. Eur J Radiol 1995;21:1-10

16. Tombach B, Heindel W. Value of 1.0- M gadolinium chelates: review of preclinical and clinical data on gadobutrol. Eur Radiol 2002;12:1550-56

17. Attenberger UI, Runge VM, Jackson CB, et al. Comparative evaluation of lesion enhancement using $1 \mathrm{M}$ gadobutrol vs. 2 conventional gadolinium chelates, all at a dose of $0.1 \mathrm{mmol} / \mathrm{kg}$, in a rat brain tumor model at $3 \mathrm{~T}$. Invest Radiol 2009; $44: 251-56$

18. Giesel FL, Mehndiratta A, Risse F, et al. Intraindividual comparison between gadopentetate dimeglumine and gadobutrol for magnetic resonance perfusion in normal brain and intracranial tumors at 3 Tesla. Acta Radiol 2009:50:521-30

19. Lemke AJ, Sander B, Balzer T, et al. Safety and use of gadobutrol in patients with brain tumors (phase III trial) [in German]. Rofo 1997;167:591-98

20. Anzalone N, Gerevini S, Scotti R, et al. Detection of cerebral metastases on magnetic resonance imaging: intraindividual comparison of gadobutrol with gadopentetate dimeglumine. Acta Radiol 2009:50:933-40

21. Thilmann O, Larsson EM, Bjorkman-Burtscher IM, et al. Comparison of contrast agents with high molarity and with weak protein binding in cerebral perfusion imaging at 3 T. J Magn Reson Imaging 2005;22:597-604

22. Benner $T$, Reimer $P$, Erb G, et al. Cerebral MR perfusion imaging: first clinical application of a $1 \mathrm{M}$ gadolinium chelate (Gadovist 1.0) in a double-blinded randomized dose-finding study. J Magn Reson Imaging 2000;12:371-80

23. Frenzel T, Lengsfeld P, Schirmer H, et al. Stability of gadolinium-based magnetic resonance imaging contrast agents in human serum at 37 degrees $\mathrm{C}$. Invest Radiol 2008;43:817-28

24. Le Duc G, Corde S, Charvet AM, et al. In vivo measurement of gadolinium concentration in a rat glioma model by monochromatic quantitative computed tomography: comparison between gadopentetate dimeglumine and gadobutrol. Invest Radiol 2004;39:385-93

25. Barker FG 2nd. Surgical and radiosurgical management of brain metastases. Surg Clin North Am 2005;85:329-45

26. Essig M, Lodemann KP, Le-Huu M, et al. Intraindividual comparison of gadobenate dimeglumine and gadobutrol for cerebral magnetic resonance perfusion imaging at $1.5 \mathrm{~T}$. Invest Radiol 2006;41:256-63

27. Gasperini C, Paolillo A, Rovaris M, et al. A comparison of the sensitivity of MRI after double- and triple-dose Gd-DTPA for detecting enhancing lesions in multiple sclerosis. Magn Reson Imaging 2000;18:761-63

28. Goyen M, Herborn CU, Vogt FM, et al. Using a 1 M Gd-chelate (gadobutrol) for total-body three-dimensional MR angiography: preliminary experience. $J$ Magn Reson Imaging 2003;17:565-71

29. Ewing JR, Brown SL, Nagaraja TN, et al. MRI measurement of change in vascular parameters in the $9 \mathrm{~L}$ rat cerebral tumor after dexamethasone administration. J Magn Reson Imaging 2008;27:1430-38 